\title{
Belphégor
}

Littérature populaire et culture médiatique

11-1 | 2013

Fantômas dans le siècle

\section{Compère, Daniel. Les romans populaires}

Paris : Les fondamentaux de la Sorbonne nouvelle, 2011. 140 p. ISBN :

978-2-87854-561-6

\section{Vittorio Frigerio}

\section{(2) OpenEdition}

Journals

Édition électronique

URL : http://journals.openedition.org/belphegor/227

DOI : 10.4000/belphegor.227

ISSN : 1499-7185

Éditeur

LPCM

\section{Référence électronique}

Vittorio Frigerio, «Compère, Daniel. Les romans populaires », Belphégor [En ligne], 11-1 | 2013, mis en ligne le 22 juin 2013, consulté le 22 septembre 2020. URL : http://journals.openedition.org/belphegor/ 227 ; DOI : https://doi.org/10.4000/belphegor.227

Ce document a été généré automatiquement le 22 septembre 2020.

\section{(c) (i) (9)}

Belphégor est mis à disposition selon les termes de la Licence Creative Commons Attribution - Pas d'Utilisation Commerciale - Pas de Modification 4.0 International. 


\section{Compère, Daniel. Les romans} populaires

Paris : Les fondamentaux de la Sorbonne nouvelle, 2011. 140 p. ISBN :

978-2-87854-561-6

\section{Vittorio Frigerio}

\section{RÉFÉRENCE}

Compère, Daniel. Les romans populaires, Paris : Les fondamentaux de la Sorbonne nouvelle, 2011. 140 p. ISBN : 978-2-87854-561-6 
Qu'on ouvrage consacré au roman populaire soit publié aux presses de la Sorbonne nouvelle montre déjà le chemin qui a été parcouru depuis un peu plus d'une vingtaine d'années dans le domaine des études sur les genres romanesques de grande diffusion. Qu'il le soit en une collection intitulée "Les fondamentaux » a de quoi réjouir ceux qui labourent depuis longtemps ce même champ. Ce qui est encore autrement plus réjouissant est qu'il y a effectivement adéquation entre le nom de la collection et les qualités de ce livre. Daniel Compère offre ici un Baedeker complet et pratique pour les voyageurs qui auraient envie de s'aventurer dans les territoires oubliés de la littérature. Il le fait de plus avec beaucoup de simplicité et de précision, sans avoir peur de tirer des conclusions là

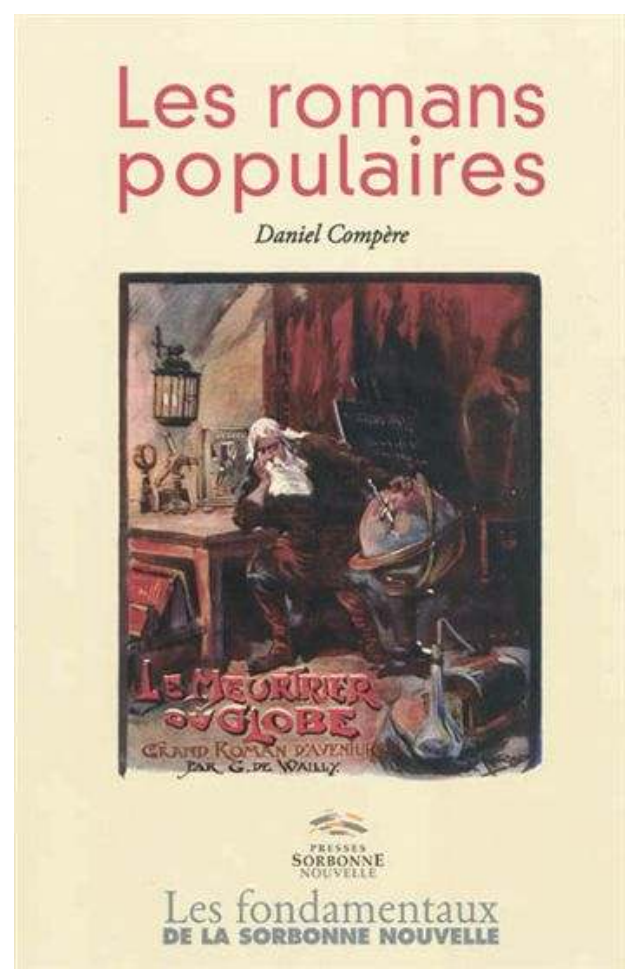
où elles s'imposent et sans avoir peur non plus de nuancer quand il le faut des affirmations communément acceptées, qui méritent pourtant d'être prises avec le grain de sel proverbial.

D'entrée de jeu, l'auteur spécifie que « comme on le verra, en dépit d'une appellation globale de "roman populaire", une grande diversité de textes se présente à nous, rappelant que chaque œuvre est singulière même si elle s'inscrit dans le projet d'un romancier, dans une collection ou dans un genre » (7). L'ouvrage entier se déroule ainsi en équilibre entre l'identification d'un certain nombre de stigmates pouvant aider à l'identification $\mathrm{du}$ "populaire » en tant que catégorie (ou pouvant du moins aider à comprendre comment il est généralement perçu), et la réaffirmation de la valeur particulière, unique et irréductible des auteurs que l'on associe généralement à ce concept.

Dans ses deux premières parties - intitulées « Un domaine aux frontières incertaines » et «Historique des romans populaires" - l'ouvrage offre un panorama historique complet, des débuts du roman populaire au dix-neuvième siècle jusqu'à l'époque contemporaine. La troisième section («Évolutions et constantes » est consacrée à une analyse de quatre pratiques littéraires identifiées comme étant spécifiques au roman populaire en tant que tel : une large diffusion; collections et séries; la pratique de la variation; l'interactivité (des lecteurs et du roman). Là encore, l'auteur évite les affirmations trop péremptoires et ne réclame pour ses catégorisations qu'une modeste valeur heuristique :

Voilà donc quelques pratiques littéraires plus ou moins développées dans les romans populaires qui permettent d'en distinguer des particularités au sein du champ littéraires. Sont-elles suffisantes pour définir le domaine des romans populaires comme véritablement différent du reste de la littérature? Nous ne le pensons pas. Elles en indiquent certainement des tendances qui sont une large diffusion sur des supports multiples, une démultiplication des intrigues, un 
développement en série, la pratique de variations et une forte interactivité. Elles permettent assurément de définir un ensemble particulier, même si les frontières avec la littérature légitimée ne sont pas nettement tracées. (71-71)

4 Armé d'une connaissance encyclopédique du sujet qu'il utilise sans la faire peser, Compère rectifie parfois le tir par rapport à certaines conceptions antérieures. Il déplace notamment le moment charnière qui voit le déclin du feuilleton et l'avènement du cinéma comme nouveau médium de masse de la Première Guerre Mondiale (comme l'affirment par exemple Jean-Claude Vareille ou Lise Dumasy), pour le mettre « à partir des années 1960 avec une forte augmentation du nombre des téléviseurs dans les foyers » (15). Il tisse également des liens ou relève des parentés qui devraient être évidentes, mais que l'histoire littéraire ne mentionne pas ou minimise. Ainsi, il identifie justement le lien qui existe entre la révolution romantique au théâtre (moment fondamental reconnu dans toutes les histoires littéraires) et la bataille du roman qui suivra peu après avec l'avènement de la presse populaire, mettant souvent en scène les mêmes acteurs, mais dont on ne trouve guère de trace dans les manuels. Au passage, s'appuyant toujours sur une connaissance de première main des textes dont il parle, il corrige des erreurs fréquemment répétées : notamment que La Vieille fille de Balzac ait été le premier feuilleton, alors qu'il a paru dans les pages internes du journal (26).

Dans la quatrième section, "Les genres principaux", le critique passe en revue, à travers quatre sous-sections divisées chacune en plusieurs sous-sous-sections, les nombreuses dénominations à travers lesquelles on a tenté, et on tente toujours, de distinguer la littérature de masse de celle institutionnalisée, qui, elle, refuserait les étiquettes. Une fois de plus, l'approche est nuancée et les typologies obtenues perdent ce qu'elles pourraient avoir de contraignant et de prescriptif chez d'autres critiques pour acquérir une utilité pratique. Ainsi est esquissé un arbre généalogique du développement des genres: "Certains genres naissent à l'intérieur d'un autre, puis prennent leur autonomie. Nous l'avons vu pour la science-fiction ou l'espionnage qui se sont progressivement détachés du roman d'aventures, qui a aussi donné naissance au roman historique et au western. Le roman d'aventures est aussi un peu à l'origine $d u$ roman policier [...]» (108). Compère ne laisse toutefois jamais les ressemblances cacher les particularités individuelles, dans lesquelles il reconnaît clairement la véritable valeur d'une littérature dont seuls ceux qui ne la connaissent pas, ni ne la fréquentent, peuvent assumer l'homogénéité: "À les regarder superficiellement, en effet, on pourrait croire que l'on retrouve des types de personnages, des situations, voire des intrigues qui se ressemblent. Mais à y regarder de près, que de différences, de nuances, de variations !» (69-70)

6 Ce livre se révèlera sans doute particulièrement utile pour ceux qui abordent pour la première fois ces questions, et devrait donc trouver naturellement sa place dans les programmes de cours consacrés au roman populaire qui apparaissent de plus en plus souvent dans les calendriers universitaires, en Europe comme ailleurs. Nombre de remarques, d'allusions, de pistes, de renvois, de conseils de lecture, vont aussi cependant stimuler les chercheurs et, c'est là du moins le souhait de l'auteur (tout comme le nôtre), les pousser à approfondir ultérieurement certaines œuvres maintenant non seulement méconnues, mais tout simplement oubliées et quasiment introuvables. Voici en tout cas un livre à la fois stimulant et agréable, aussi grâce à quelques pointes d'humour discret, telle la suivante, avec laquelle nous concluons cette recension en laissant la parole à l'auteur : 
La logique de l'institution scolaire a longtemps été de mettre en avant la notion d'œuvre et surtout de chef-d'œuvre, excluant ainsi la majeure partie des textes publiés. Ici aussi se met en place un principe de reproduction: les enseignants d'aujourd'hui sont les élèves d'hier et ils étudient avec leurs élèves ce qu'euxmêmes ont étudié. Heureusement que les sciences ne s'enseignent pas comme les lettres... (121)

\section{AUTEURS}

\section{VITTORIO FRIGERIO}

Université de Dalhousie 